\title{
Influence des perturbations anthropiques sur les communautés de petits mammifères des savanes gabonaises
}

\author{
Jean-François MBOUMBA ${ }^{1,2^{*}}$, Brice Roxan MOMBOUA ${ }^{1}$, Eline PERIN ${ }^{2}$ et \\ Fréderic YSNEL ${ }^{2}$
}
IDépartement de Biologie, Université des Sciences et Techniques de Masuku, Franceville B.P. 901, Gabon.
${ }^{2}$ Université de Rennes 1- MNHN, UMR BOREA, 35042 Rennes cedex, France.
*Auteur correspondant ; E-mail: jeanfrancois.mboumba@univ-masuku.org ; mboumbafr@yahoo.fr

\begin{tabular}{lll}
\hline Received: 26-02-2021 & Accepted: 11-08-2021 & Published: 31-08-2021 \\
\hline
\end{tabular}

\section{RESUME}

Les savanes gabonaises connaissent des feux anthropiques récurrents, entraînant des dommages sur cet écosystème. L'influence de ce facteur sur la diversité des micromammifères n'a jamais été étudiée. Une situation préoccupante pour ce taxon qui fait l'objet de très peu de programmes de protection. A travers l'analyse de la distribution spatiale des espèces (Muridés), l'étude vise à mesurer l'empreinte écologique des activités anthropiques dans trois régions du Gabon, présentant des niveaux de perturbations différents. Au total 259 individus appartenant à six espèces de muridés ont été capturés avec un effort de 6220 nuit-pièges. Les rendements de capture varient de 0,5 à $15 \%$. Mus minutoides (62,5\%) et Lemniscomys striatus (28.9\%) sont les plus abondantes. La richesse spécifique (S) varie de 1 à 4 espèces dans les savanes post -feu, alors qu'elle est de 5 dans les savanes protégées des feux, et de 2 espèces dans les savanes fauchées puis brulées. La diversité la plus élevée a été observée dans les savanes non exposées au feu $\left(\mathrm{H}^{\prime}=1,10 ; \mathrm{J}=0,68\right)$. Ceci atteste que le feu influe sur l'abondance, et lorsqu'il est préalablement associé à la fauche, il agirait à la fois sur la diversité et sur l'abondance d'espèces locales.

(C) 2021 International Formulae Group. All rights reserved.

Mots clés : Feux de brousse, communautés des Muridés, diversité, petits rongeurs tropicaux, Gabon.

\section{Influence of human disturbance to the small mammal communities in the Gabonese savannahs}

\begin{abstract}
In the issue of the use of recurrent fires in Gabon, we carried out an inventory both in the Gabonese savannahs that are regularly burned and in those that are not. The study was carried out in the southern and central savannahs of Gabon during the periods from 2004, 2012 and 2013. A total of 259 individuals representing six species of small savannah rodents were captured over 6,220 trap nights. Trap success was variable (0.5 to $15 \%)$. Mus minutoides (62.5\%) and Lemniscomys striatus (28.9\%) are the most abundant. Small rodent abundance was significantly higher in the savannahs protected from fires. The species richness varies from 1 to 4 species in the
\end{abstract}


post-fire savannahs and up to 5 species in the savannahs protected from fires. The savannahs that were mowing prior to burning have yielded only 2 species. The highest diversity index ( $\left.\mathrm{H}^{\prime}\right)$ is found in savannahs not exposed to fire $\left(H^{\prime}=1.10\right)$. Bush fires seem to have a direct effect on species abundance. Conversely, when savannahs are mown prior to burning, both the diversity and abundance of local species communities are affected. This study gave an initial idea of the diversity of the rodent population in this the recurrent use to fires.

(C) 2021 International Formulae Group. All rights reserved.

Keywords : Savannah fires, Muridae communities, diversity, small tropical rodents, Gabon.

\section{INTRODUCTION}

Les petits mammifères constituent un groupe faunistique dont la protection des espèces est encore assez négligée dans le Bassin du Congo (Afrique Centrale) et notamment au Gabon. L'état de conservation de la plupart des espèces est mal connu et par conséquent, elles font l'objet de très peu de programmes de protection (Denys et al., 2014). Pourtant, elles jouent un rôle vital dans le fonctionnement et la régénération des écosystèmes (Denys et al., 2014 ; Hauptfleisch et al., 2017). Leurs capacités à coloniser différents milieux naturels et anthropisés (Happold, 2013), et à réagir rapidement aux changements de leur environnement (Avenant et Cavallini, 2007), en font des bons indicateurs écologiques pertinents pour évaluer l'intensité des perturbations pouvant être induites par les pressions anthropiques dans les habitats naturels. La majorité des études d'inventaires des communautés de petits mammifères en Afrique centrale, notamment au Gabon ont été effectuées principalement dans les forêts (Nicolas et Colyn, 2003 ; O’Brien et al., 2006 ; Morgan et al., 2020). Alors que la littérature en lien avec la diversité de petits mammifères forestiers ne cesse d'augmenter, celle de petits mammifères savanicoles du Gabon reste encore pauvrement documentée, nonobstant une forte présence des savanes $(15 \%$ du territoire) (Walter et al., 2005 ; Maloba Makanga, 2011). Implantées tantôt au sein du massif forestier Guinéo-Congolais (savanes intra forestières) plus ou moins isolées : cas des savanes de la Lopé (Mboumba et al., 2020), tantôt en périphérie orientale et australe du massif forestier (cas des savanes des Plateaux Batéké, de la Ngounié et de la N'yanga dans la vallée du Niari), cette diversité de savanes créé des conditions favorables à l'établissement des communautés de petits mammifères. Malheureusement, l'anthropisation de ces habitats naturels par différents patterns de perturbations (feux, aménagement, écotourisme, fauche, agriculture, chasse, braconnage, etc.) est assez récurrente au Gabon comme partout en Afrique Subsaharienne (N'Dri et al., 2018). Généralement les feux de brousse sont considérés comme le facteur de perturbation anthropique le plus récurrent dans ces milieux savanicoles (Valea et Ballouche, 2012). C'est un facteur écologique important, qui influe sur la diversité des espèces. Depuis 2002, le Gabon a entrepris une série d'action dont la création d'un réseau de 13 Parcs Nationaux (PN) et des aires protégées (Peyrot, 2008) s'étendant sur plus de $10 \%$ du territoire, représentatif de l'ensemble des écosystèmes du pays et consacrés à la conservation de la biodiversité. Parallèlement, la quasi-totalité des savanes gabonaises sont représentées dans ce réseau national, et demeurent donc protégées dans leur diversité. Cependant, tous les ans, des vastes étendues savanicoles situées à l'extérieur comme à l'intérieur des zones protégées sont soumises à des feux de brousse (Valea et Ballouche, 2012; Bergström et al., 2018), et dans une moindre mesure, à l'action conjuguée de la fauche et du feu de brousse (cas de la Lékédi). En définitif, dans ce contexte culturel dans lequel le brûlage se produit tous les ans, les populations autochtones et gestionnaires de l'environnement, intègrent le feu dans la gestion de la savane. Aujourd'hui, les feux de brousse ont une motivation écologique : conserver les savanes en leur état, et maintenir ou accroître les populations des grands 
mammifères inféodés aux milieux savanicoles (Christy et Wilmé, 2003).

Des études ont récemment montré que le brûlage local peut être bénéfique pour la gestion des ressources (Laris, 2002 ; Christy et Wilmé, 2003). En ce sens, pour les gestionnaires des parcs, l'utilisation appropriée des feux est devenue un véritable outil de gestion et de valorisation des paysages savanicoles (Dadem et al., 2018). Cette pratique traditionnelle (Valea et Ballouche, 2012) du feu de brousse annuel est itératif et anarchique au Gabon comme dans tous les pays d'Afrique centrale et de l'ouest (Valea et Ballouche, 2012 ; N'Dri et al., 2018). Elle est considérée depuis longtemps comme un «moindre mal». Ces feux, qui sont majoritairement présents au cours des mois de la saison sèche (juillet et août), sont essentiellement d'origine anthropique. Les objectifs des feux diffèrent sensiblement. Le feu de brousse est couramment utilisé en savane pour : la chasse collective (Christy et Wilmé, 2003), le braconnage (Kabore, 2010), faciliter les déplacements des villageois, l'aménagement des savanes (PN), la conversation des ilots et des étendues savanicoles. Il est aussi utilisé pour la diminution des herbacées afin de stimuler la repousse végétale (Ghebrehiwot et al., 2011) favorable aux grands herbivores. La récurrence de feux (planifiés ou non) sur la végétation ligneuse peut avoir des répercussions sur la gestion de la faune sauvage, surtout lorsqu'il s'étend sur d'importantes superficies (Christy et Wilmé, 2003). Cela est sans doute vrai pour les petits mammifères comme pour le reste de la faune au sol (amphibiens, insectes, etc.) qui ne pourraient rejoindre un abri pour se protéger (Christy et Wilmé, 2003). On peut donc s'attendre à ce que le régime de gestion des savanes puisse modifier la structure des communautés de ce groupe en créant des conditions environnementales locales spécifiques. Toutefois, il a été démontré, que les différents niveaux de perturbations des milieux naturels peuvent être appréciés sur la base des modifications observées dans leurs compositions fauniques originelles (Gentili et al., 2014). En effet, la diversité et l'abondance des micromammifères peuvent être considérées comme des paramètres écologiques capables d'aider à l'appréciation de l'empreinte écologique des activités humaines dans les milieux naturels (Men et al., 2015).

Récemment, en Afrique de l'Est, des études ont montré que le feu et le butinage postincendies auraient des effets néfastes sur les populations locales des petits mammifères dans les écosystèmes savanicoles (Bergström et al., 2018). C'est dans ce contexte habituel des feux au Gabon, en l'absence d'évaluation périodique et d'indicateurs spécifiques, que les rongeurs ont été choisis, pour illustrer les conséquences éventuelles des perturbations anthropiques parmi les petits mammifères. Dans le cadre d'un programme mené sur la distribution à grande échelle spatiale de petits rongeurs savanicoles du Gabon. L'étude est une contribution à la connaissance de la biodiversité de ce taxon. Plus spécifiquement, il s'agit d'une analyse comparative de la composition et de la structure des communautés de rongeurs, inventoriés dans trois régions qui diffèrent par leur degré de perturbation. Pour cela, l'étude s'est fixée pour objectif principal de : déterminer la diversité des communautés des espèces de petits rongeurs (Muridés) à la fois dans les savanes post-feu dans deux régions distinctes (Plateaux Batéké et Lopé) et dans une savane protégée des feux dans la région de la N'yanga ; et comparer la structure et la composition des communautés à l'intérieur d'une même region (échelle locale) d'une part, et d'autre part, entre les localités des différentes régions (échelle régionale). Cela aboutira à une meilleure connaissance de l'influence du feu de brousse sur la biodiversité de petits rongeurs à l'échelle locale et régionale.

\section{MATERIEL ET METHODES}

\section{Sites d'étude \\ Les savanes des Plateaux Batéké}

Les savanes des Plateaux Batéké sont localisées dans le sud-est du Gabon (Figure 1) et sont maintenues par des feux réguliers d'origine anthropique (Walter et al., 2005). La végétation est dominée par la présence des 
forêts primaires et secondaires, par une mosaïque de savanes herbeuses (à Hyparrhenia diplandra et Pobeguinea arrecta) et arbustives (dominées par Hymenocardia acida et Annona senegalensis), entrecoupées des forêts galeries humides constituées d'essences diverses. Ici, la flore herbeuse est plus pauvre (plus de détails lire Mboumba et al., 2020). C'est une zone de fortes précipitations par rapport à une grande partie du sud-ouest du Gabon. Cependant, l'échantillonnage s'est déroulé à Franceville en mai 2004 sur les deux rives du fleuve Ogooué (plus grand fleuve du Gabon) : rive droite (FRD : S0132' 43.0" ; E132 29' 27.0") et la rive

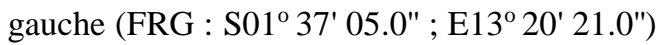
au sein des savanes post-brulis dans la localité de Mbaya. En complément de données dans cette région, en 2012 et 2013, un focus a été réalisé dans les savanes du parc privé de la

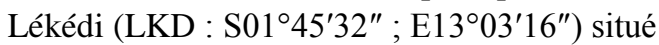
à Bakoumba (à $100 \mathrm{~km}$ de Franceville), afin de mesurer l'influence du double effet conjugué, $\mathrm{du}$ feu et de la fauche. Ce parc est entièrement clos et occupe 14000 hectares divisé en trois modules fermés et inégalement répartis. Le paysage est dominé par de forêts primaires et secondaires avec une mosaïque forêt-savane bien représentée. Dans ce site, l'échantillonnage s'est déroulé dans les modules 1 et 2. Dans ces derniers, les patterns de pertubation sont principalement la fauche et les feux d'aménagement des savanes, contrôlés par les agents chargés de l'entretien du parc. Les zones de savanes choisies pour cette étude, sont celles qui ont été brûlées un à deux ans avant ce travail. La campagne de piégeage a été réalisée en fin juin et début juillet 2012, puis de mi-avril à début mai 2013.

\section{Les savanes de la Lopé}

Les savanes de Lopé sont localisées dans le centre du Gabon à l'intérieur du large bloc forestier Guinéo-Congolais. Le paysage est constitué d'une mosaïque de forêts et de savanes à graminées (Christy et Wilmé, 2003) montrant des paysages très diversifiés. Ces savanes présentent une faible richesse végétale. Elles abritent de nombreuses espèces d'herbes fréquemment rencontrées en Afrique centrale, comme Pobeguinea arrecta, Hyparrhenia diplandra, Anedelphia arrecta, Nauclea latifolia. La Lopé se trouve dans une zone de faibles précipitations par rapport à une grande partie du Gabon (1500 mm par an). L'échantillonnage a eu lieu début mai 2004, dans un premier temps dans deux savanes postfeu du Parc National de la Lopé (brûlées 1 à 2 ans auparavant ; PNL : S00 04' 00" ; E11 ${ }^{\circ} 34^{\prime}$ $00^{\prime \prime)}$. Dans ce parc, les patterns de pertubation sont principalement les feux annuels (juillet et août) d'aménagement des savanes programmés par le gestionnaire et son personnel. On a été également échantillonné dans la zone tampon situé à $20 \mathrm{~km}$ au sud-est du PNL, dans les savanes post-feu (brulées 1 à 2 ans avant cette étude) de la localité de Kazamabika (KZM : S00 $08^{\prime} 10.0^{\prime \prime}$; E11 $\left.{ }^{\circ} 42^{\prime} 00^{\prime \prime}\right)$. Dans ce site, les feux de brousse gérés par les populations locales et l'agriculture sont la principale menace.

\section{Savanes de la N'yanga}

Les savanes de la N'yanga localisées dans le sud du Gabon, constituent deux corridors séparés par la forêt. Elles sont principalement herbeuses avec quelques espèces d'arbustes qui sont très variés dans la plupart des régions d'Afrique soudanozambézienne (Dupont et al., 2000). Les arbustes sont des espèces pionnières très résistantes au feu. Les espèces dominantes dans la plupart de ces savanes sont : Hymenocardia acida, Anonna arenaria, Bridelia ferruginea, Sarcocephalus esculentus (Mboumba et al., 2020). L'étude s'est déroulée en Avril 2004 dans la localité de Mourindi (MRD : S 02 ${ }^{\circ} 16^{\prime}$ $00^{\prime \prime}$; E $\left.10^{\circ} 30^{\prime} 00^{\prime \prime}\right)$ où les savanes sont largement réprésentées. Cette localité est située dans la zone tampon du Parc National de Moukalaba-Doudou (PNMD), localisé au sudouest du pays. Il est caractérisé par une mosaïque de paysage : forêt de montage, forêt primaire et secondaire, parfois interrompues par des petites mosaïques de savanes. La zone d'étude est un secteur de vaste savane incluse non perturbée par les activités humaines.

\section{Choix des modèles}

Les modèles retenus, sont les rongeurs de la famille des muridés. Du fait de leur large valence écologique, plusieurs espèces de ce taxon présentent une large distribution dans les 
savanes africaines. Elles sont abondantes dans les savanes et se reproduisent toute l'année (Denys et al., 2014). Par ailleurs elles ne sont pas impactées par les activités de chasses locales. Ces caractéristiques communes à la plupart des espèces de muridés leur confèrent donc un rôle bioindicateur pour les études comparatives d'impacts, aussi bien liés à la gestion locale qu'à large échelle spatiale.

\section{Méthode de capture et d'identification des espèces}

La technique utilisée est la ligne de piégeage (transect). Pour minimiser les biais introduits par différentes techniques de piégeage, trois types de pièges ont été utilisés (dans l'ensemble des zones d'études) : pitfalls (pièges à trou utilisant des seaux en plastique de $10 \mathrm{~L})$, pièges Sherman $(7,5 \times 9 \times 23 \mathrm{~cm})$, ces deux dispositifs permettent la capture vivant des spécimens, et enfin, des pièges à ressort (10 x $15 \mathrm{~cm}$; Mboumba et al., 2020). L'unité de piégeage, est une ligne de 100 mètres (20 seaux), pour les pitfalls, et $250 \mathrm{~m}$ pour les deux autres types de pièges, qui correspond à 50 pièges ( 25 Sherman et 25 à ressorts), posés en alternance et espacés de 5 mètres (Mboumba et al., 2020). Chaque localité dispose de 150 pièges actifs qui correspondent à trois lignes de pièges, et de 40 à 60 seaux, soit trois lignes de pitfalls maximums. Si plus de $20 \%$ des captures au jour 4 étaient de nouvelles captures, le piégeage était prolongé jusqu'à une sixième ou septième nuit. A l'inverse, le piégeage était suspendu si après quatre nuits, $100 \%$ des captures étaient des individus des espèces déjà capturées. Afin de favoriser l'appétence, et diminuer le biais d'échantillonnage lié au choix : la noix de palme et le tubercule de manioc sont utilisés comme appât (Mboumba et al., 2020). Après la capture des spécimens, la majorité des individus de petits rongeurs ont été euthanasiés par dislocation cervicale. Cette étape était nécessaire à l'identification spécifique des individus à partir de l'étude de leurs caractéristiques morphologiques externes. Les identifications ont été réalisées sur le terrain à l'aide de la clef d'identification et des ouvrages de références (Kingdon, 2003; Happold,
2013), puis confirmées par des analyses moléculaires pour certains individus. Les spécimens ont ensuite été conservés en formol.

\section{Analyses statistiques}

Les différents peuplements des communautés de petits rongeurs ont été caractérisés à l'aide des indices d'abondance relative et de diversité. Pour évaluer les différences entre les compositions et les structures des peuplements des communautés, l'analyse des données a été réalisée à l'aide des packages pgirmess R (Giraudoux et al., 2018) et vegan. Auparavant, le calcul du succès de piégeage $(\mathrm{T})$ ou rendement de piégeage a été effectué. Ce dernier est un indice d'abondance relative (Avenant et Cavallini, 2007) qui est basé sur le nombre d'individus capturés par 100 nuits de piégeage. Cet indice a été calculé pour les différentes savanes étudiées. Il est calculé comme suit : $(\mathrm{T})=[(\mathrm{Nm} / \mathrm{NTN}) \times 100]$ où $\mathrm{Nm}$ est le nombre total d'individus et NTN est le nombre de nuits de piégeage (Kadjo et al., 2013). Par la suite, les indices de diversité ont été calculés pour chaque localité. La richesse spécifique (S) calculée, correspond au nombre total d'espèces échantillonné dans un habitat donné (Ofori et al., 2016). L'abondance relative $\left(P_{i}\right)$ qui est l'expression de l'importance de chacune des espèces par rapport à toutes celles enregistrées dans un habitat a été calculée à l'aide de la formule suivante $: P_{i}=(\mathrm{ni} / \mathrm{N}) \times 100 ;$ avec $\mathrm{ni}=$ nombre d'individus de l'espèce $\mathrm{i} ; \mathrm{N}=$ nombre total de spécimens capturés. Il a été également, utilisé deux autres indices pour caractériser la diversité au sein de différentes communautés : les Shannon-Weaver $\left(\mathrm{H}^{\prime}\right)$ et de régularité ou d'équipartition de Pielou (J). L'indice de diversité $\left(\mathrm{H}^{\prime}\right)$ a été calculé à l'aide de la formule $: \mathrm{H}^{\prime}=-\sum$ pi $\mathrm{x} \ln (p i)$; où pi est le rapport entre le nombre d'individus d'espèces particulières et le nombre total d'individus capturés. L'indice de régularité $(\mathrm{J})$ a été calculé à l'aide de la formule : $\mathrm{J}=\mathrm{H}^{\prime} / \ln (\mathrm{S})$, où $\mathrm{H}^{\prime}$ est le nombre dérivé de l'indice de diversité de Shannon et $\mathrm{S}$ est la richesse des espèces (CISSE et al., 2020). Elle indique, l'équipartition de l'abondance de chaque espèce dans le peuplement des communautés. 
Les valeurs vont de 0 à 1 . Plus la valeur est élevée, plus la communauté est équilibrée (Kouadio et al., 2018). La normalité des données a été vérifiée au préalable par un test Shapiro-Wilk. Le test de Kruskal-Wallis (KW) a permis de comparer les abondances relatives des différentes espèces échantillonnées. Pour apprécier le niveau de différence du succès de piégeage entre les savanes des trois régions, un test de khi2 basé sur l'effort de piégeage et sur les effectifs des individus d'espèces capturées a été calculé. Le même test a été appliqué entre les savanes de FCV et de LKD dans lesquelles sont pratiquées à la fois la fauche et les feux de brousse, afin d'arborer l'interaction de ces deux facteurs. Les analyses ont été réalisées avec le logiciel R (version 3.5.2).

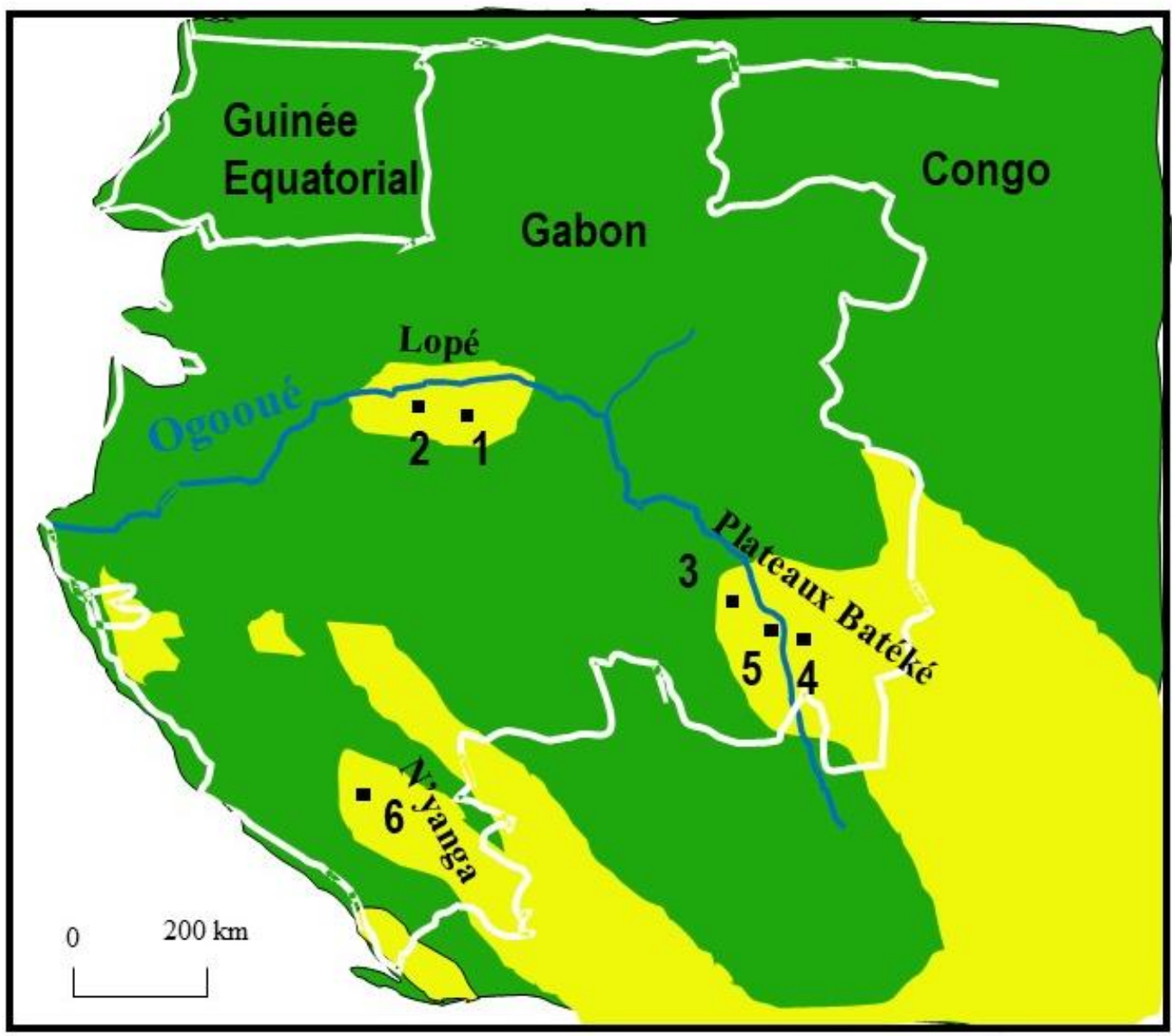

Forêts

\section{Savanes}

Figure 1 : Carte des sites d'études (figure modifiée à partir de celle de Mboumba et al., 2020). Savanes de la Lopé (centre Gabon) : (1) Kazamabika (KZM), (2) Parc National de la Lopé (PNL) ; Savanes des Plateaux Batéké : (3) Parc privé de la Lékédi (LKD), (4) Franceville rive droite (FRD), (5) Franceville rive gauche (FRG) ; les savanes de la N'yanga (zone tampon de Moukalaba-Doudou): (6) Mourindi (MRD). 


\section{RESULTATS \\ Composition des communautés de petits rongeurs}

Au total 249 individus ont été capturés en 4300 nuits pièges pour l'ensemble des sites en 2004, ce qui représente un succès de piégeage (T) moyen de 5.8\%. Six espèces appartenant à la famille des muridés constituent la communauté locale de rongeurs (Tableau 1). De manière globale, le succès de piégeage varie de 2.8 à $15.42 \%$. A l'échelle locale, il est très faible dans les savanes post brulis de FCV (2.8\%) malgré un effort de piégeage important (1800 NP), de 4 à $5 \%$ dans les savanes post brûlis de la Lopé pour un effort de piégeage moins important ( $900 \mathrm{NP}$; Table 1). A l'inverse, dans les savanes de la N'yanga (MRD), qui ne sont pas affectées par la pratique régulière du feu de brousse (Table 2), le succès de piégeage est de $15 \%$ pour un effort de piégeage de $700 \mathrm{NP}$ Par ailleurs, la composition des espèces diffère également en fonction des régions et des localités (Table 1). Mus minutoides et Lemniscomys striatus ont été collectés dans toutes les savanes régionales et représentent respectivement $65 \%$ et $29 \%$ des captures. Les plus rares sont Lophuromys sikapusi (5\%) et Pelomys campanae (2.7\%), suivies de Dendromus cf. mystacalis $(0.38 \%)$ et de Mastomys natalensis (0.38\%). A l'échelle locale, Mus minutoides est présente dans cinq localités, à l'inverse $L$. striatus et $L$. sikapusi sont présentes dans quatre et trois localités respectivement (Table 1).

\section{Richesse spécifique, comparaison des diversités et des abondances relatives}

Dans l'ensemble, la richesse spécifique est faible et diffère à l'intérieur d'une même région (cas de la Lopé). Dans les savanes de FCV, sur 4 espèces capturées, deux sont dominantes : M. minutoides et $L$. striatus avec des abondances relatives de 30 à 50 (Table 1). Elle est également faible dans les savanes de la Lopé (KZM et PNL) où 4 espèces ont été capturées (Table 1), et une seule domine les peuplements: M. minutoides avec des abondances relatives de 30 à $100 \%$. La différence entre les deux peuplements porte sur 3 espèces, présentes dans la zone tampon (KZM) et absentes dans le parc. A l'inverse, dans les savanes de la N'yanga (MRD), 5 espèces ont été capturées, dont deux déjà signalées dans les Plateaux Batéké dominent le peuplement (abondances à plus de $40 \%$; Table 1). Pour les autres espèces, les abondances n'accèdent pas $10 \%$ quel que soit la localité (Table 1). Dans ces habitats, M. munitoides domine L. striatus (Table 1). De façon générale, les indices de Shannon-Weaver $\left(\mathrm{H}^{\prime}\right)$ sont faibles. Cependant, la plus forte valeur est enregistrée à MRD $\left(\mathrm{H}^{\prime}=1.10\right)$, et décroit légèrement dans les savanes post-feu de $\mathrm{FCV}$ $\left(\mathrm{H}^{\prime}=1.01\right)$, puis elle est inférieure à 0,5 à KZM et nul dans le PNL. (Table1). L'équitabilité (J) est élevée dans les savanes de FCV et de MRD $(\mathrm{J}=0.73$ et $\mathrm{J}=0.68$; Table 1$)$. En revanche, il est plus faible à KZM. Le test de KruskalWallis (KW) révèle que les abondances relatives interspécifiques varient significativement d'une savane à l'autre (KW $=9.026$, Df $=3, \mathrm{p}<0.0289$ ). Bien que les abondances relatives interspécifiques soient significativement différentes, l'analyse de Box-plot montre qu'il n'y a pas de différence significative entre les abondances de $L$. striatus et de M. minutoides (Figure 2). Le test du Khi2 appliqué sur l'effort de piégeage et les effectifs d'individus capturés, montre une différence significative entre les régions $\left(\chi^{2}=285.13, \mathrm{df}\right.$ $=3, \mathrm{p}<0,001)$.

Par ailleurs, en complément de l'étude mené en 2004, dans les Plateaux Batéké (FCV), il ne faut cependant pas perdre de vue les résultats obtenus 2012/2013 (qui ont nécessité un effort de piégeage de 1920 NP avec un total des 10 individus capturés ; Table 1), dans le cas particulier du parc de la Lékédi (LKD) où sont pratiqués la fauche et le feu de brousse. De façon inattendue, malgré son statut de conservation, l'étude révèle que les indices de diversité de petits rongeurs sont faibles dans cette zone. En effet, les données montrent que le succès de piégeage est 5 à 30 fois inférieur comparativement aux autres zones de 2004 (Table 1), et ce malgré un effort de piégeage relativement important (1920 NP). Le focus fait à LKD révèle une faible diversité spécifique, deux espèces ont été capturées, et une domine le peuplement (Table 1). A l'échelle des Plateaux Batéké, les abondances moyennes entre les échantillonnages effectués sur FCV en 2004 vs LKD en 2012/2013 varient significativement $\left(\chi^{2}=30.8, \mathrm{df}=1, \mathrm{p}<0,001\right)$. 
Table 1 : Diversité et abondance relative de petits rongeurs dans trois régions savanicoles aux patterns des perturbations anthropiques différents.

\begin{tabular}{|c|c|c|c|c|c|c|c|c|c|c|c|}
\hline \multirow{3}{*}{$\begin{array}{l}\text { Regions } \\
\text { Codes localités } \\
\text { Especes }\end{array}$} & \multicolumn{4}{|c|}{ Plateaux Batéké } & \multicolumn{4}{|c|}{ Lopé } & \multicolumn{2}{|c|}{ N'yanga } & \multirow{3}{*}{ Total } \\
\hline & \multicolumn{2}{|c|}{$\begin{array}{c}\text { LKD } \\
(N P=1920)\end{array}$} & \multicolumn{2}{|c|}{$\begin{array}{c}\text { FCV } \\
(N P=1800)\end{array}$} & \multicolumn{2}{|c|}{$\begin{array}{c}\text { PNL } \\
(N P=900)\end{array}$} & \multicolumn{2}{|c|}{$\begin{array}{c}\text { KZM } \\
(N P=900)\end{array}$} & \multicolumn{2}{|c|}{$\begin{array}{c}\text { MRD } \\
(\mathrm{NP}=700)\end{array}$} & \\
\hline & capture & $\mathrm{Pi}(\%)$ & capture & $\operatorname{Pi}(\%)$ & capture & $\operatorname{Pi}(\%)$ & capture & $\mathrm{Pi}(\%)$ & capture & $\mathrm{Pi}(\%)$ & \\
\hline Dendromus cf. mystacalis (Heuglin, 1863) & 0 & 0 & 0 & 0 & 0 & 0 & 0 & 0 & 1 & 0,92 & 1 \\
\hline Lemniscomys striatus (Linneaus, 1758) & 9 & 90 & 19 & 37,25 & 0 & 0 & 3 & 6,98 & 44 & 40,74 & 75 \\
\hline Lophuromys sikapusi (Temminck, 1853) & 0 & 0 & 5 & 9,8 & 0 & 0 & 1 & 2,3 & 7 & 6,48 & 13 \\
\hline Mastomys natalensis (Smith, 1834) & 0 & 0 & 0 & 0 & 0 & 0 & 1 & 2,3 & 0 & 0 & 1 \\
\hline Pelomys campanae (Huet, 1888) & 0 & 0 & 1 & 1,96 & 0 & 0 & 0 & 0 & 6 & 5,55 & 7 \\
\hline Mus minutoides (Smith, 1834) & 1 & 10 & 26 & 50,98 & 47 & 100 & 38 & 88,4 & 50 & 46,29 & 162 \\
\hline Total & 10 & 100 & 51 & 100 & 47 & 100 & 43 & 100 & 108 & 100 & 259 \\
\hline Succès de piégeage (T \%) & \multicolumn{2}{|c|}{0,5} & \multicolumn{2}{|c|}{2,83} & \multicolumn{2}{|c|}{5,22} & \multicolumn{2}{|c|}{4,77} & \multicolumn{2}{|c|}{15,42} & \\
\hline \multicolumn{12}{|l|}{ Indice de diversité } \\
\hline Nombre d'espèces (S) & \multicolumn{2}{|c|}{2} & \multicolumn{2}{|c|}{4} & \multicolumn{2}{|c|}{1} & \multicolumn{2}{|c|}{4} & \multicolumn{2}{|c|}{5} & \\
\hline Shannon-Weaver (H') & \multicolumn{2}{|c|}{0.32} & \multicolumn{2}{|c|}{1.01} & \multicolumn{2}{|c|}{0} & \multicolumn{2}{|c|}{0.46} & \multicolumn{2}{|c|}{1.10} & \\
\hline Equitabilité (J) & \multicolumn{2}{|c|}{0.47} & \multicolumn{2}{|c|}{0.73} & \multicolumn{2}{|c|}{0} & \multicolumn{2}{|c|}{0.33} & \multicolumn{2}{|c|}{0.68} & \\
\hline
\end{tabular}

Nombre des spécimens capturés, succès de piégeage $(\mathrm{T})$, indices de diversité $\left(\mathrm{S}, \mathrm{H}^{\prime}\right.$ et $\left.\mathrm{J}\right)$, abondance relative (Pi) et effort de piégeage (NP). Compte tenu du faible nombre de capture dans LKD, nous avons cumulé les effectifs obtenus sur les deux saisons d'échantillonnage (2012 et 2013) dans ce site.

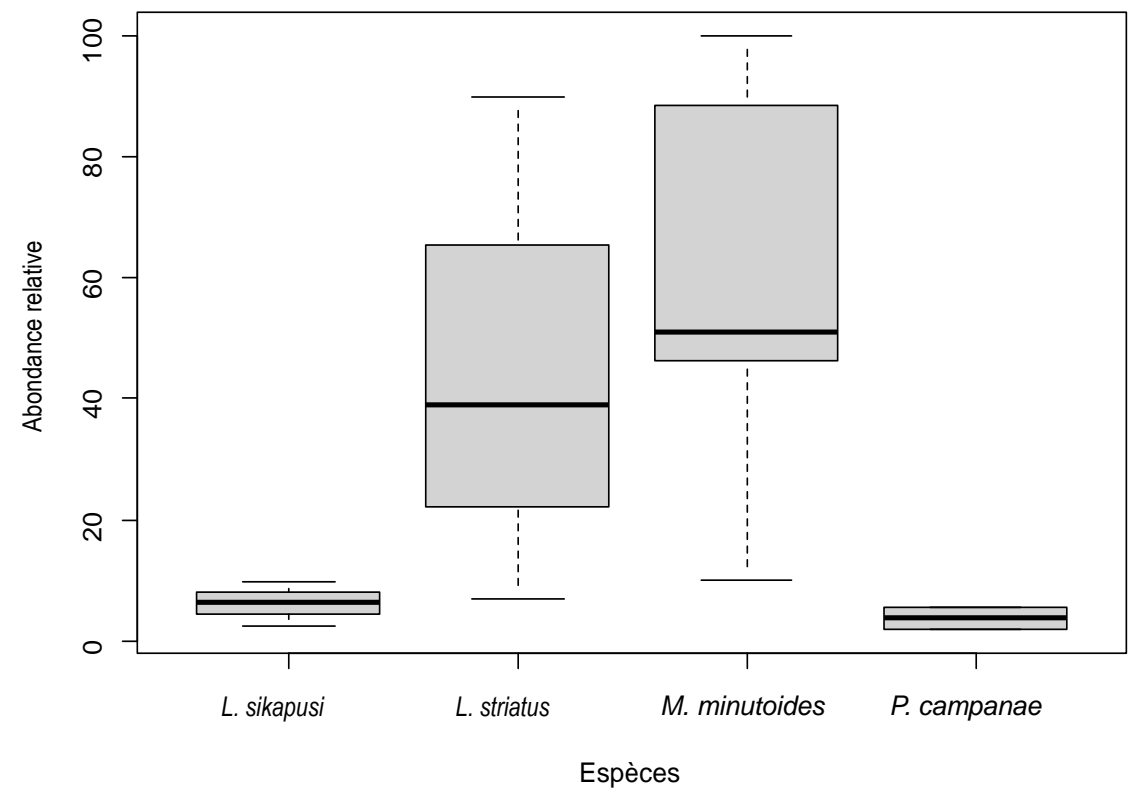

Figure 2 : Box-plot des abondances relatives en fonction des espèces et des savanes échantillonnées. 


\section{DISCUSSION}

A l'échelle régionale, six espèces typiques des savanes africaines ont été capturées, et sont généralement communes aux trois régions. La capture de Mus minutoides, Lemniscomys striatus et Lophuromys sikapusi dans les savanes des trois régions prospectées témoigne de leur grande capacité d'adaptation aux modifications du milieu. M. minutoides et $L$. striatus sont les plus constantes, elles supportent mieux les perturbations naturelles et anthropiques régnant dans les trois régions. Elles s'adaptent facilement aux différents types de succession végétale. Le spectre de répartition régionale des espèces met en évidence deux gradients écologiques de répartition opposés : i) un gradient sud-nord négatif caractérisé par une forte abondance de L. striatus dans les savanes de MRD, qui diminue en proportion à KZM et qui disparait à $\mathrm{PNL}$. Il en est de même pour L. sikapusi qui devient de plus en plus rare à KZM et inexistant à PNL ; ii) un gradient sud-nord positif, avec M. minutoides qui au contraire, est de plus en plus abondant à la Lopé. La présence d'une espèce à affinité australe (Pelomys campanae) connue des savanes zambéziennes, a été également capturée dans les savanes du sud (FCV et MRD). Ce résultat pourrait attester d'un ancien couloir savanicole persistant entre les savanes gabonaises et les savanes zambéziennes qui se maintiendrait potentiellement par l'action annuelle des brulis. Ce résultat est en accord avec Maley (2001). L'effort de piégeage varient significativement d'un secteur à l'autre. Il a été plus élevé dans les savanes post brulis des Plateaux Batéké et de la Lopé, où les pressions humaines sont omniprésentes. Malgré cela, les rendements ont été assez faibles dans ces régions. Ces résultats pourraient s'expliquer par différentes formes d'anthropisations. Avec, un effet direct sur la reconstitution extrêmement lente des peuplements de petits rongeurs. Partant de ce constat, l'étude montre l'importance d'une végétation haute afin de conserver une certaine diversité. Selon
Maisonneuve et Rioux (1998) l'abondance des micromammifères augmenterait graduellement avec l'étagement de la végétation. Les résultats, laissent à penser de l'hétérogénéité de la couverture végétale post-feu, pourraient entrainer un biais dans le rendement. Il faut savoir que, lors des feux de brousse, la végétation brûle de manière inégale, ce qui détermine une mosaïque de zones denses non brûlées (résidus de végétation de l'année précédente) et de zones claires (tapis végétaux de l'année en cours). Par conséquent, selon l'étendue de la surface de ces zones males brûlées, on pourrait s'attendre à un rendement hétérogène des captures.

A l'échelle locale, la présence de Mastomys natalensis dans les savanes de la zone tampon (KZM) du PNL est une marque d'anthropisation. En effet, les pressions humaines qui règnent dans la zone de KZM antérieures à cette étude, auraient favorisé l'établissement de $M$. natalensis. Selon Duplantier et Granjon (1988), cette espèce préfère les champs et les milieux perturbés. La faune murine du PNL est gravement diminuée au regard de celle de sa zone tampon à KZM, avec une seule espèce commune capturée. Par ailleurs, la présence de Dendromys cf. mystacalis (espèce arboricole) dans les savanes de MRD est assez surprenante. Elle est connue des savanes du nord-est du Gabon uniquement (Dubost, 1965). Cet auteur a décrit les limites géographiques de sa localisation que dans le nord-est. Le fait que cette espèce soit capturée au-delà de sa zone de répartition supposée, et à l'aide des pièges qui ont été posés au sol interpelle. Cette présence, provient-elle d'une colonisation récente corrélée aux pratiques de gestion des savanes de MRD ? Cela semble peu probable, car cette espèce tout comme la majorité des espèces de petits rongeurs strictement savanicoles et à large distribution, sont incapables de traverser la forêt. Ce qui voudrait dire, que vu l'isolement géographique entre MRD et les savanes du nord-est (séparées par un grand manteau forestier), il est certainement probable que cette espèce 
(strictement savanicole) se soit établie dans le sud. Il semble néanmoins, que sa présence dans ces savanes implique que l'espèce préfère les savanes protégées des feux.

L'appréciation des différentes perturbations anthropiques des savanes ne saurait se limiter qu'aux seuls effets du feu. Il a paru utile de faire un focus sur les savanes post-feu de la LKD où le feu est associé à la fauche régulièrement. Les résultats obtenus montrent deux fois moins d'espèces à l'intérieur du parc de LKD comparer à FCV (ou à KZM). Si ce n'est pas un effet du décalage temporel important entre les périodes d'échantillonnage, ce résultat pourrait suggérer que l'action combinée de la fauche et du feu accroît l'influence des perturbations anthropiques sur le peuplement local de petits rongeurs, en modifiant profondément la richesse spécifique à comparer à celle maintenue par le feu uniquement (cas FCV et KZM). Il parait probable, que lors de la fauche (par des machines), de nombreux petits rongeurs soient décimés (cas des juvéniles nidicoles notamment), ce qui semble favoriser un faible renouvellement des populations locales. A noter également, bien que les mêmes techniques de collecte aient été appliquées, ce contre-coup pourrait avoir une tout autre explication, qui serait liée au décalage de la cyclicité des abondances des espèces pendant la période d'étude. Toutefois, on reste très prudent sur cette dernière hypothèse. Cependant, ce résultat est également en accord avec Hauptfleisch (2014) qui a montré que l'abondance des petits mammifères était plus élevée dans les zones non fauchées que dans les zones fauchées. Ce même résultat suppose aussi, que les communautés de petits rongeurs ont des exigences plus importantes en termes de couverture végétale avec un faible pourcentage de sol nu.

De façon globale, on peut donc s'interroger sur l'absence des espèces à large distribution (L. striatus, L. sikapusi et $P$. campanae) dans le PNL (voire LKD). Dans ce cas, il apparait vraisemblablement que les populations locales de ces espèces sont en grande partie décimées par les modes de gestion. Ces absences, ne peuvent avoir une corrélation, ni avec la biogéographie des espèces, dans la mesure où elles sont déjà présentes dans ces régions et au niveau des savanes voisines, ni avec la saisonnalité des feux (juillet/août), puisque globalement elle ne change pas d'une localité à l'autre. On peut donc supposer une relation directe avec les pratiques de gestion, qui créeraient des conditions locales spécifiques. En revanche, les espèces non rencontrées à l'intérieur des parcs (PNL ou LKD) sont-elles des marqueurs d'un environnement local, perturbé par des méthodes de gestion ? Cependant, il convient de s'interroger sur ces méthodes compte tenu des enjeux, en termes de maintien de la biodiversité dans ces parcs. Il est possible aussi, qu'elles n'ont pas été capturées malgré les efforts consentis ? Ce non renouvellement d'une population locale est éventuellement explicable par une faible fécondité dû au stress, ou à leur capacité de récupérer après une perturbation dans des conditions de faible croissance démographique (en particulier quand la productivité des milieux est faible), c'est vrai pour les savanes du PNL, et c'est d'autant plus vrai pour celles de LKD. Ces inconvénients pourraient contribuer à troubler la recoloniser des habitats naturels post-feu, en raison de la disparition de la litière et de la haute végétation. Probablement aussi, par leur manque de propension à la recolonisation des milieux perturbés, corrélée à la faible densité globale du peuplement. Le site de LKD illustre bien cette observation. Ce constat de la fluctuation des espèces de petits rongeurs selon le niveau de perturbation de leur habitat naturel, n'a jamais été étudié au Gabon. Par conséquent, on n'a pas assez de recul (absence des travaux de références), permettant toutes comparaisons et interprétations cohérentes des résultats obtenus pour conclure, bien que la faune murine soit bien impactée. 
Les résultats de la composition des peuplements présentent des signatures différentes. Il est dominé par deux espèces $(M$. minutoides et L. striatus; Figure 2), suivi de deux espèces peu représentées (L. sikapusi et Pelomys campanae) en proportions variables suivant les localités. Les indices de diversité et d'équitabilité des communautés reflètent les intensités de perturbation supposées, et les valeurs les plus élevées correspondent aux secteurs les moins perturbés (région de la N'yanga, MRD). Les valeurs de ces indices peuvent cependant différer entre les savanes à l'intérieur d'une même région. Ce résultat pourrait suggérer que les pratiques locales de gestion engendrent des effets spécifiques. On note des diversités (et des abondances) beaucoup plus faibles à l'intérieur des parcs (PNL à LKD) comparativement aux zones de savanes situées en périphérie et au-delà. Ce résultat est en accord avec ceux de Caro (2001) et de Muck and Zeller (2006) qui ont montré que l'abondance et la diversité des petits mammifères étaient plus élevées dans les zones adjacentes aux aires protégées qu'à l'intérieur de ces aires. Caro (2001) a émis l'hypothèse que la concurrence interspécifique entre les ongulés qui broutent continuellement et les petits mammifères, ainsi que la réduction de la couverture par le pâturage des grands herbivores pourraient impacter les communautés de rongeurs. Si le peuplement des petits rongeurs apparait plus équilibré dans les savanes de la N'yanga et des Plateaux Batéké, que dans la zone de Lopé (KZM), la réponse est autre, il y a une à deux espèces qui dominent les peuplements (M. minutoides et $L$. striatus). La dominance par une poignée d'espèces (Figure 2), démontre que, plus le milieu semble impacté par l'anthropisation, et plus la présence de petits rongeurs est faible. A l'échelle locale, il ressort un fait important de cette analyse de la structure des peuplements des communautés de petits rongeurs : la redondance des profils de la structure des communautés. Dans les savanes post-feu où, le feu de brousse est la seule principale pertubation (FCV, PNL et KZM), le profil est dominé par M. minutoides, suivi de L. striatus. Ce même profil est retrouvé dans les savanes marquées par une absence de pression humaine (MRD), mais avec une grande nuance sur les proportions de ces deux espèces. Ces proportions sont largement supérieures dans les savanes de MRD que dans toutes les autres savanes. Ce résultat semble témoigner que les patterns de perturbation et les modes de gestions humains sont, pour tout ou partie importante, la cause de la dégradation de la structure des communautés locales de petits rongeurs. Un tout autre profil, qui diffère du précédent est observé dans les savanes où l'activité anthropique principale (malgré un intervalle temps), se résume par une succession de fauche suivie par des feux de brousse (LKD), ce dernier est dominé par L. striatus. Les activités humaines, surtout la superposition de la fauche et du feu de brousse, influencent fortement la structure du peuplement de communautés de petits rongeurs. Le feu de brousse semble agir directement sur les abondances relatives des espèces. A l'inverse, lorsqu'il est préalablement associé à la fauche, il agirait à la fois sur la richesse spécifique et sur les abondances relatives des espèces de petits rongeurs (cas de LKD).

\section{Conclusion}

La diversité spécifique du peuplement de petits rongeurs vivant dans les savanes postfeu du Gabon est potentiellement estimée à partir des résultats de piégeage. À ces patterns de pertubation, correspond une certaine diversité spécifique du peuplement de rongeurs provisoirement représentée par 6 espèces strictement savanicoles (et à large échelle de distribution). L'impact de l'anthropisation a été ressenti par la présence d'un individu de l'espèce Mastomys natalensis capturé à KZM. Une autre espèce, L. sikapusi évite les savanes situées à l'intérieur des parcs alors que deux espèces murines (M. minutoides et $L$. striatus) sont corrélées aux savanes des trois régions étudiées. En somme, les résultats illustrent 
l'intérêt des études d'analyses sommaires de la composition du peuplement pour inférer l'état des habitats à partir des espèces mammaliennes, dès le moment qu'elles sont strictement inféodées à un type de paysage. L'étude donne un aperçu général sur la composition des communautés de petits rongeurs des savanes gabonaises, postérieurement à l'action du feu de brousse, et dans une moindre mesure sous l'action de la fauche. Il a semblé utile de commencer par une approche superficielle à grande échelle de l'occurrence des feux de brousse ou d'aménagement des savanes. L'étude a pu utilement apporter un éclairage sur l'évolution de la composition du peuplement des muridés, afin de se focaliser ensuite sur des zones d'intérêts liées au maintien de la biodiversité (PNL et LKD) qui montrent une faible diversité murine. Cependant, des études comparatives approfondies (avec un effort accru) complémentaires à l'échelle locale devraient être entreprises à l'intérieure du PNL ou LKD entre parcelles post-feu et non brûlées, pour permettre d'affiner la connaissance de l'évolution de la composition du peuplement (ou des sous peuplements) des muridés en fonction des perturbations anthropiques.

\section{CONFLIT D'INTERETS}

Les auteurs de cet article déclarent qu'il y a aucun conflit d'intérêts.

\section{CONTRIBUTIONS DES AUTEURS}

Travail a été réalisé à $80 \%$ par JFM, $10 \%$ par les étudiants et $10 \%$ par FY.

\section{REMERCIEMENTS}

Nous remercions le directeur de la SODEPAL (Parc de la LKD) pour avoir fourni le cadre et la logistique. Nous remercions également l'Université des Sciences et Techniques de Masuku (USTM) qui a mis en place ce partenariat avec la SODEPAL. Nos remerciements vont aussi à la Direction de la Faune et de la Chasse (Ministère des Eaux et Forêt du Gabon) pour leur aide apportée sur le plan administratif (permis de recherche), et logistique dans les zones de la Lopé et de Moukalaba Doudou (Mourindi). Nous remercions également le Professeur Denis Webb pour sa relecture du résumé en anglais.

\section{REFERENCES}

Benzécri JP, Benzecri F. 1982. L'Analyse des Données (Tomes I \& II). Dunod : Paris ; p.632.

Bergström BJ, Sensenig RL, Augustine DJ, Young TP. 2018. Searching for cover : soil enrichment and herbivore exclusion, not fire, enhance African savanna smallmammal abundance. Ecosphère, 9(11). www.esajournals.org

Caro TM. 2001. Species richness and abundance of small mammals inside and outside an African national park. Biol. Conserv., 98: 251-257.

CISSE A, OUATTARA M, N'GUESSAN EA, N'Gouan ABROU JE. 2020. Diversité végétale et usages des plantes dans une zone de savane soudanienne : Cas de la localité de Ferkessédougou (Nord, Côte d'Ivoire). Int. J. Biol. Chem. Sci., 14(8): 2807-2825.

Christy P, Wilmé L. 2003. Lopé, un almanach équatorial. Ecofac, Libreville-Gabon. p.95.

Dadem Gueguim C, Tchamba NM, Fotso CR. 2018. Dynamique spatio-temporelle des feux de brousse dans le Parc National du Mbam et Djerem (Cameroun). Int. J. Biol. Chem. Sci., 12(2): 728-748.

Denys C, Missoup AD, Nicolas V, Fülling O, Delapré A, Bilong Bilong CF, Taylor PJJ, Hutterer R. 2014. African highlands as mammal diversity hotspots: new records of Lamottemys okuensis Petter, 1986 (Rodentia: Muridae) and other endemic rodents from $\mathrm{Mt} \mathrm{Oku}$, Cameroon. Zoosystema, 36(3): 647-690.

Dubost G. 1965. Un Muridé arboricole du Gabon, Dendromus pumilio Wagner, possesseur d'un cinquième orteil opposable. Biol. Gabonica, 1 :187-190. 
Duplantier JM, Granjon L. 1998. Occupation et utilisation de l'espace par des populations du genre Mastomys au Sénégal : étude de trois niveaux de perception. Sciences et Techniques des animaux de Laboratoire, 13 : 129-131.

Dupont LM, Jahns S, Marret F and Ning S. 2000. Vegetation change in equatorial West Africa: time-slices for the last 150 ka. Palaeo., 155: 95-122.

Gentili S, Sigura M, Bonesi L. 2014. Decreased small mammals species diversity and increased population abundance along a gradient of agricultural intensification. Italian-Journal-of Mammalogy, 25(1): 39-44.

Ghebrehiwot HM, Kulkarni MG, Light ME, Kirkman KP, Van Staden J. 2011, Germination activity of smoke residues in soils following a fire, South African Journal of Botany, 77 :718-724.

Giraudoux P, Antonietti JP, Beale C, Pleydell D, Treglia M. 2018. Spatial Analysis and Data Mining for Field Ecologists. URL: http://perso.orange.fr/giraudoux

Guillotin M, Duplantier JM. 1982. Richesses comparées des peuplements en Guyane et au Gabon. Série Zoologique, 132 : 85-89.

Happold DCD. 2013. Rodents, hares and rabbits. In: (J. Kingdon, D.C.D Happold, M. Hoffmann, T. Butynski, M. Happold and J. Kalina, eds.) Mammals of Africa. London Bloomsbury Publishing. pp. 9121696.

Hauptfleisch ML. 2014. The use of ecosystem parameters in predicting the risk of aircraft-wildlife collisions at Namibian airports. PhD Thesis, University of the Free State, Bloemfontein, 256p.

Hauptfleisch ML, Vinte MP, Blaum N. 2017. A comparison of the community dynamics of bioturbating small mammals between live stock and Wild life farming areas in the Kalahari, Namibia, Vol 1. Section A. pp. 34-39.
Kabore A. 2010. Brousse des uns, aire protégée des autres Histoire du peuplement, perceptions de la nature et politique des aires protégées dans le Gourma burkinabè: l'exemple de la Réserve partielle de faune de Pâma ». Thèse de l'institut de Hautes Etudes Internationales et du Développement. Genève, 386p.

Kouadio YJC. Soiret KSP, Kpan WB, Yao NO, N'guessan KE, Kouassi KP, Gomez PJ. 2018. Valeur de conservation de la Réserve Naturelle Volontaire (RNV) de la Dodo, Sud-Ouest de la Côte d'Ivoire (Afrique de l'Ouest). Int. J. Biol. Chem. Sci., 12: 2784-2796.

Laris P. 2002. Burning the seasonal mosaic: preventative burning strategies in the wooded savanna of southern Mali. Human Ecology, 30(2): 155-186.

Maley J. 2001. La destruction catastrophique des forêts d'Afrique centrale survenue il y a environ 2500 ans exerce encore une influence majeure sur la répartition actuelle des formations végétales. Syst. Geogr. Pl., 71: 777-796.

Maloba Makanga J.D. 2011. Mosaïque forêtsavane et exploitation des ressources forestières du Gabon. Geo-Eco-Trop. 35 : 41-50.

Mboumba JF, Hervé MR, Guyot V, Ysnel F. 2020. Small rodent communities (Muridae) in Gabonese savannas: species diversity and biogeographical affinities. Mammalia, 1-13.

Men XY, Guo XG, Dong WG, Ding, N, \& Qian TJ. 2015. Influence of Human Disturbance to the Small Mammal Communities in the Forests. Open Journal of Forestry, 5 : 1-9.

Moisonneuve C, Rioux S. 1998. Influence de l'étagement de la végétation dans les bandes riveraines en milieu agricole sur leur utilisation par les micromammifères et l'herpétofaune. Ministère de l'Environnement et de la Faune du Québec. Direction de la faune et des habitats. p.57. 
Morgan K, Mboumba JF, Ntie S, Mickala P, Miller CA, Zhen Y, Harrigan RJ, Le Underwood V, Ruegg K, Fokam EB, Tasse Taboue GC, Sesink Clee PR, Fuller T, Smith TB and Anthony NM. 2020. Precipitation and vegetation shape patterns of genomic and craniometric variation in the central African rodent Praomys misonnei. Proc. R. Soc. B, p.10.

Muck C, Zeller U. 2006. Small mammal communities on cattle and game grazing areas in Namibia. African Zoology, 41 : 215-223.

N'Dri AB, Fongbe M, Soro TD, Gignoux J, Kone M, Dosso K, N'Dri JK, Kone NA, Barot S. 2018. Principaux indices de l'intensité du feu dans une savane Guinéenne d'Afrique de l'Ouest. Int. J. Biol. Chem. Sci., 12(1) : 266-274.

Nicolas V, Colyn M. 2003. Seasonal variations in population and community structure of small rodents in a tropical forest of Gabon. Can. J. Zool., 81: 1034-1046.

O'Brien C., McShea W, Guimondou S, Barrière P, Carleton M. 2006. Petits mammifères terrestres (Soricidés et Muridés) du Complexe d'Aires Protégées de Gamba, Gabon: composition taxinomique et comparaison de méthodes d'échantillonnage. Bull. Biol. Soc. Wash., 12: $137-148$.
Ofori BY, Attuquayefio DK, Owusu EH, Musah Y, Ntiamoa-Baidu Y. 2016. Spatio-temporal variation in small mammal species richness, relative abundance and body mass reveal changes in a coastal wetland ecosystem in Ghana. Environmental Monitoring and Assessment, 188(330) : 1-10.

Peyrot B. 2008. Incidences écologiques, anthropologiques et paléoécologiques sur l'évolution des forêts du Gabon. Les Cahiers d'Outre-Mer, Revue de Géographie de Bordeaux, LXI : 111-144. Sousa WP. 1984. The role of Disturbance in Natural communities. Ann. Rev. Ecol. Syst., 15 : 353-391.

Valea F, Ballouche A. 2012. Les feux de brousse en Afrique de l'Ouest : contraintes environnementales ou outil de gestion environnementale? L'exemple du Burkina Faso. Territoires d'Afrique, 3 : 36-47.

Waters G, Bradley A, Niangadouma R. 2005. Floristics of Gabon's Batéké Plateaux: Guineo Congolian plants on Kalahari Sands. In African Plants: Biodiversity, Ecology, Phytogeography and Taxonomy, Ghazanfar SA, Beentje H (eds). Royal Botanic Gardens : Kew. 